\title{
Outcome in patients with large intraventricular haemorrhages: a volumetric study
}

\author{
Y B W E M Roos, D Hasan, $M$ Vermeulen
}

\begin{abstract}
Patients with large amounts of intraventricular blood have a poor prognosis. The question is whether the quantity of ventricular blood or other factors related to the origin of the intraventricular bleeding cause poor outcome.

The association between ventricular blood volume and outcome was analysed in patients with a primary intraventricular haemorrhage (PIVH) and in patients with an aneurysmal subarachnoid haemorrhage (SAH). The medical records of 24 patients with PIVH were reviewed and these patients were compared with 59 patients selected from a prospectively studied series of patients with aneurysmal SAH who had intraventricular blood without an intraparenchymal haematoma. Glasgow coma score on admission and outcome at three months expressed on the Glasgow outcome scale were available for all patients. Volumetric analysis on CT was carried out if there was at least a moderate amount of blood measured with a semiquantitative scale (SAH $n=30$, PIVH $\mathrm{n}=22$ ).
\end{abstract}

In patients with SAH more than $20 \mathrm{ml}$ intraventricular blood was lethal, as previously shown for patients with intraparenchymal haemorrhage. Patients with a PIVH can, however, survive such large volumes of intraventricular blood without impaired consciousness immediately after the bleeding, without developing hydrocephalus, and without any disability after three months.

It is concluded that the cause of an intraventricular haemorrhage is more important for outcome than the amount of intraventricular blood, and large intraventricular haemorrhages are not always associated with hydrocephalus.

(F Neurol Neurosurg Psychiatry 1995;58:622-624)

Ziekenhuis Rotterdam

Dijkzigt, The

Netherlands

D Hasan

Correspondence to:

Dr Yvo Roos, Department of Neurology, Academic Medical Center (AMC), Meibergdreef $9,1105 \mathrm{AZ}$ Amsterdam zuidoost, The Netherlands.

Received 20 June 1994 and in final revised form 3 January 1995

Accepted 10 January 1995 patients with intraparenchymal or subarachnoid haemorrhage. ${ }^{12}$ In a series of patients with supratentorial haemorrhage all patients with more than $20 \mathrm{ml}$ of ventricular blood died except one, who remained in a vegetative state. ${ }^{1}$ The question is whether ventricular blood or other factors cause poor outcome. The finding that patients with a primary intraventricular haemorrhage (PIVH) may survive without neurological impairment at least suggests that the adverse outcome is not always caused by the presence of large amounts of blood in the ventricles. ${ }^{34}$ Volumetric analysis of ventricular blood has never been carried out, however, in a group of patients with PIVH.

The aim of this study was to investigate whether a large haemorrhage $(>20 \mathrm{ml})$ in the ventricles causes poor outcome. Therefore we investigated the association between ventricular blood volume and outcome in patients with PIVH and in patients with aneurysmal subarachnoid haemorrhage (SAH). In patients with PIVH the intraventricular haemorrhage is the main determinant of outcome whereas in patients with aneurysmal SAH many factors - such as rebleeding, cerebral ischaemia, hydrocephalus, and operation-are related to outcome. The results were compared with those of a previous published series of patients with ventricular haemorrhage and an intracerebral haematoma. ${ }^{1}$ We also determined whether the level of consciousness on admission predicts the cause of the intraventricular haemorrhage.

\section{Patients and methods}

We reviewed the medical records of all patients registered between 1983 and 1990 at the Dijkzigt University hospital with a diagnosis of non-traumatic non-aneurysmal intracranial haemorrhage. From this series we selected patients with a primary intraventricular haemorrhage (PIVH) - that is, an intracranial haemorrhage without a bleeding pattern compatible with ruptured aneurysm and without parenchymal blood (blood limited to the ventricular cavities).$^{4}$ If a small quantity of blood was present in the subarachnoid space in those patients, it was never located in the basal cisterns, the frontal interhemispheric fissure, or the sylvian fissure. These patients with PIVH were compared with patients with an aneurysmal haemorrhage pattern on their initial CT, with intraventricular blood but without an intraparenchymal haematoma. ${ }^{5}$ 
These patients with SAH were selected from a prospectively studied consecutive series of patients from the same centre with aneurysmal SAH admitted between 1984 and 1988. All of these patients received standard care including hypervolaemia without antihypertensive treatment. None was treated with nimodipine and only 10 patients had antifibrinolytic treatment (four days).

In all patients (SAH and PIVH) CT had been performed within 72 hours after the onset of the haemorrhage. The clinical condition of all patients on admission had been recorded on the Glasgow coma scale. Outcome at three months after the haemorrhage was available for all patients and was assessed with the Glasgow outcome scale.

The amount of intraventricular blood was graded as previously described. ${ }^{5}$ If the score was at least 2 in one of the lateral ventricles, volumetric analysis was carried out as described by Young et al. ${ }^{1}$ In each slice containing haemorrhage we measured the surface of the intraventricular haemorrhage in arbitrary units. This surface was then converted to $\mathrm{cm}^{2}$ from a scale printed on each scan. Finally each calculated area was multiplied by scan slice thickness and the values were summated to derive ventricular volumes for each haemorrhage.

\section{Results}

In 28 of the 59 patients with an aneurysmal bleeding pattern on the initial CT an aneurysm as the bleeding source was proved by angiography or at necropsy. Eight patients had an aneurysm of the anterior communicating artery, seven of the posterior circulation, five of the posterior communicating artery, four of the middle cerebral artery, two of the pericallosal artery, one of the ophthalmic artery and one of the carotid artery. The aneurysm was treated surgically in 19 patients, on average 17 (range 10-28) days after the initial haemorrhage. Four of these patients died and in two of these there was a surgical complication.

Of the remaining 40 patients-who were not operated on-35 died, 15 as a direct effect of the initial haemorrhage, 13 of rebleedings, one of cerebral ischaemia, and one of hydrocephalus. Three patients died of extracranial complications and in two patients the cause of death could not be established.

Of 751 patients with a non-traumatic, nonaneurysmal, intracranial haemorrhage, 24 (3.2\%) patients had a PIVH. In 14 of these patients angiography was carried out; eight had an arteriovenous malformation and one a posterior communicating aneurysm, probably not related to the haemorrhage because there was no blood present in the subarachnoid space. In five patients angiography was normal. One arteriovenous malformation was found at necropsy. In 10 patients angiography was not carried out; in six this was because of death very soon after admission, five of these as a direct effect of the initial haemorrhage and one because of a cardiopulmonary com- plication. In the other four patients angiography was not performed as surgery was not considered. This was because these patients remained in a very poor clinical condition (two) or because of old age (two).

The mean age was similar for the groups of 24 patients with PIVH and 59 patients with SAH (PIVH 53 (range 7-79) years and SAH 52 (range 22-81) years). The Glasgow coma score on admission was also similar between these two groups. Patients with PIVH more often had a large intraventricular haemorrhage than patients with $\mathrm{SAH} ; 20(83 \%)$ of 24 patients with PIVH had a volume of more than 6 on the semiquantitative scale $v 29$ (49\%) of 59 patients with SAH. (95\% confidence interval of the observed difference between these percentages $14 \cdot 6 \%-53 \cdot 8 \%$.)

The table lists the main characteristics of those patients with PIVH or SAH in whom volumetric analysis was performed and who had at least a moderate amount of ventricular blood on the semiquantitative scale (at least 2 in one of the lateral ventricles). Age and Glasgow coma score on admission were similar between these two selected patient groups. Sixteen $(73 \%)$ of 22 patients with PIVH had a ventricular blood volume of $20 \mathrm{ml}$ or more compared with $11(37 \%)$ of 30 patients with $\mathrm{SAH}$. None of the 11 patients with $\mathrm{SAH}$ with ventricular blood volume of $20 \mathrm{ml}$ or more was in a good clinical state shortly after the initial haemorrhage (Glasgow coma score 13 or 14). By contrast, five of the 16 patients with PIVH and a ventricular blood volume of over $20 \mathrm{ml}$ had a coma score of 13 or 14 on admission.

The figure shows that all patients with $\mathrm{SAH}$ and a volume of over $20 \mathrm{ml}$ of ventricular blood had died at three months. No such lethal volume was found for patients with PIVH. The age of patients with SAH and a ventricular blood volume of over $20 \mathrm{ml}$ varied between 36 and 79 (mean 58) years. The age of patients with PIVH who had volumes of over $20 \mathrm{ml}$ of ventricular blood and died varied between 43 and 79 (mean 65) years; and those who survived were between 14 and 74 (mean 47) years.

In the total group, eight patients with a ventricular blood volume of over $20 \mathrm{ml}$ survived

Characteristics of patients in whom volumetric analysis was performed

\begin{tabular}{lcc}
\hline & $\begin{array}{l}S A H \\
n=30\end{array}$ & $\begin{array}{l}P I V H \\
n=22\end{array}$ \\
\hline $\begin{array}{l}\text { Female } \\
\text { Male }\end{array}$ & $15(50)$ & $9(41)$ \\
Age (mean range) & $15(50)$ & $13(59)$ \\
Amount of intraventricular & $53(27-81)$ & $53(7-79)$ \\
blood & & \\
on CT & & \\
$\quad 0-10$ & $11(37)$ & $1(5)$ \\
$11-20$ & $8(27)$ & $5(23)$ \\
$21-30$ & $3(10)$ & $7(32)$ \\
$>30$ & $8(27)$ & $9(41)$ \\
GCS on admission: & $10(33)$ & $8(36)$ \\
$\quad 13-14$ & $9(30)$ & $6(27)$ \\
$8-12$ & $11(37)$ & $8(36)$ \\
$<8$ &
\end{tabular}

Values, apart from age, are numbers of patients (\%) 
A

Subarachnoid haemorrhage $(n=30)$

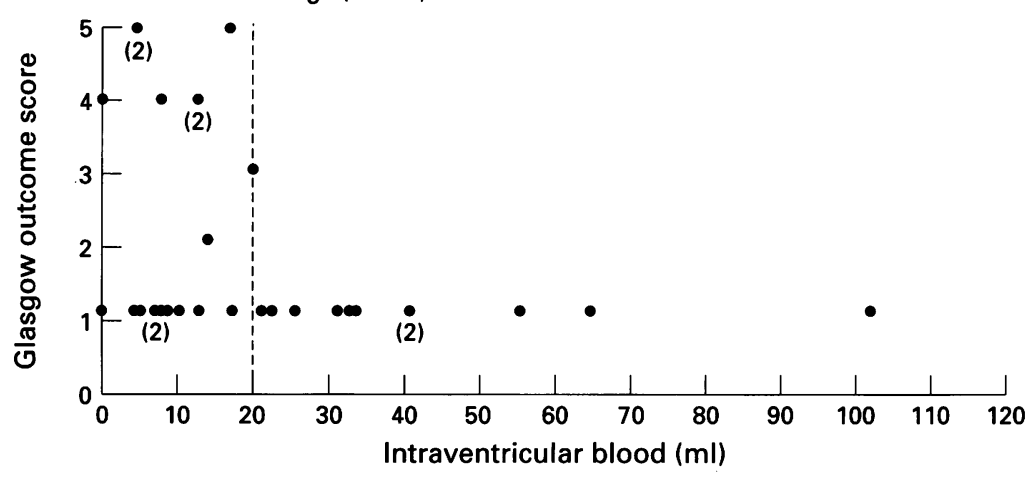

B

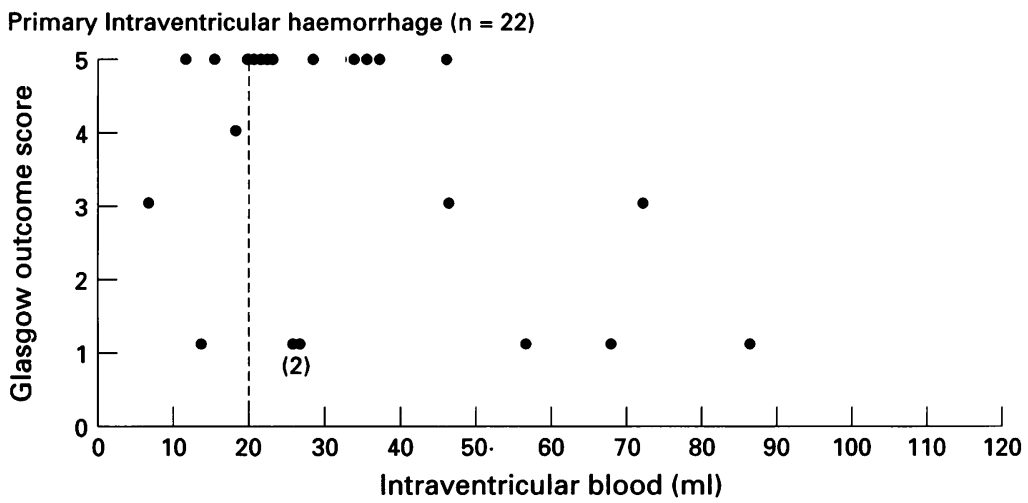

Association between Glasgow outcome score (1 = death; 2 = persistent vegetative state; $3=$ severe disability; $4=$ moderate disability; $5=$ good recovery) and intraventricular blood volumes in $(A)$ subarachnoid haemorrhage and $(B)$ primary intraventricular haemorrhage. ness and poor outcome in patients with large haemorrhages is probably the sharp rise in intracranial pressure at the time of bleeding, resulting in ischaemic damage. The lethal volume of $20 \mathrm{ml}$ ventricular blood in patients with intraparenchymal haemorrhage or SAH is apparently an expression of the severity of the haemorrhage. It is therefore unlikely that in these patients operative removal or fibrinolytic treatment of the intraventricular blood clot with tissue plasminogen activator would result in improvement of outcome. ${ }^{6}$

Our second question was whether the level of consciousness on admission predicts the aetiology of the intraventricular haemorrhage in the absence of an intraparenchymal haematoma. Therefore we compared patients with PIVH with patients with SAH who were matched for equally large amounts of ventricular blood. We found no significant difference in the level of consciousness on admission between these groups. Very large amounts of blood, however, more than $20 \mathrm{ml}$, were always associated with impaired consciousness in patients with aneurysmal SAH by contrast with patients with a PIVH who could have unimpaired levels of consciousness even in the presence of more than $20 \mathrm{ml}$ intraventricular blood. Therefore in patients with very large intraventricular haemorrhages and a normal or near normal level of consciousness (Glasgow coma score 13 or 14) an aneurysm as the cause of the bleeding is unlikely.

A conspicuous finding was that spontaneous recovery without drainage is possible in the presence of large volumes of intraventricular blood in patients with PIVH. A further question is whether ventricular drainage is indicated in this group of patients. Outcome may be excellent without drainage and these patients showed that even large intraventricular haemorrhages are not always associated with hydrocephalus. This might mean that hydrocephalus does not develop in patients with large intraventricular bleedings as long as there is no blood in the basal cisterns. On the other hand small amounts of blood, even in the perimesencephalic cisterns, may be enough to cause hydrocephalus.

In conclusion, factors other than the quantity of intraventricular blood determine outcome in patients with intracranial haemorrhage, and large intraventricular haemorrhages are not always associated with hydrocephalus.

1 Young WB, Lee KP, Pessin MS, Kwan ES, Rand WM, Caplan LR. Prognostic significance of ventricular blood in supratentorial hemorrhage: A volumetric study. Neurology 1990;40:616-9.

2 Hijdra A, Van Gijn J, Nagelkerke NJD, Vermeulen $M$, bral ischemia and poor outcome after aneurysmal subbral ischemia and poor outcome after aneurysmal
arachnoid hemorrhage. Stroke 1988;19:1250-6.

3 Darby DG, Donnan GA, Saling MA, Walsh KW, Bladin PF. Primary intraventricular hemorrhage: clinical and PF. Primary intraventricular hemorrhage: clinical and
neuropsychological findings in a prospective stroke neuropsychological findings in
series. Neurology 1988;38:68-75.

4 Caplan LR. Primary intraventricular hemorrhage. In: Kase CS, Caplan LR, eds. Intracerebral hemorrhage. London: Butterworth-Heinemann, 1994:383-401

5 Hijdra A, Brouwers PJAM, Vermeulen M, Van Gijn J. Grading the amount of blood on computed tomograms of the subarachnoid hemorrhage. Stroke 1990;21: 1156-1161.

6 Findlay JM, Grace MGA, Weir BKA. Treatment of intraventricular hemorrhage with tissue plasminogen activator. Neurosurgery 1993;32:941-7. ischaemia, hydrocephalus, and surgical complications. The cause of impaired conscious- 